\title{
Crianças com dificuldade de aprendizagem e a escola: emoções e saúde em foco
}

\author{
Learning disability children and the school: \\ the emotions and the health in focus
}

\author{
Sônia Regina Fiorim ENUMO \\ Erika da Silva FERRÃO² \\ Mylena Pinto Lima RIBEIRO3
}

\begin{abstract}
Resumo
Relações entre emoções e saúde de alunos com e sem dificuldade de aprendizagem foram analisadas por indicadores de ansiedade, depressão, stress, doenças físicas, queixas somáticas, faltas escolares, peso e altura. Responderam ao Inventário de Ansiedade Traço-Estado para Criança e à Escala de Stress Infantil 60 alunos (8-14 anos) da 3a e 4a série, divididos em G1 (desempenho inferior no Teste de Desempenho Escolar) e G2 (desempenho superior no Teste de Desempenho Escolar). A Escala de Sintomatologia Depressiva para Mestres e o questionário sobre doenças e queixas somáticas dos alunos foram respondidos por 6 professoras. Não houve diferenças significativas entre dados das escalas, mas naqueles obtidos com as professoras, identificando-se 23,3\% da amostra com indicações de risco para problemas emocionais e de saúde. Formaram-se dois subgrupos - G1a e G2a - com 14 alunos, entrevistados individualmente sobre o estado de saúde e a escola, dados esses submetidos à análise funcional, identificando-se eventos do contexto escolar e familiar que dificultam o desempenho acadêmico.
\end{abstract}

Palavras-chave: dificuldade de aprendizagem; estado emocional; estudantes; saúde.

\begin{abstract}
Relation between emotions and health for the students with and without learning disabilities have been analyzed, using anxiety, depression, stress, physical diseases, somatic complaints, school absences, weight and height indicators. Sixty students (8-14 years old), attending 3rd and 4th grades, divided in G1 (low performance in a Test of Academic School) and G2 (high performance), have answered the IDATE-C and the Child Stress Symptoms Inventory. Six teachers answered the Symtomatology of Depression Scale for Teachers and the students' diseases and somatic complaints questionnaire. There were no significant difference the group data scale, however, the interview data had identified 23.3\% of the sample presented risk of emotional and health problems. Two groups were formed - G1 a (learning disabilities) and G2a-composed by 14 students, who have been interviewed individually and, according to a data functional analysis about their health condition and the school, presented school and family experiences that hamper their academic performance.
\end{abstract}

Key words: learning disabilities; emotional states; students; health.

\section{$\operatorname{rove}$}

1 Professora Doutora, Programa de Pós-Graduação em Psicologia, Centro de Ciências Humanas e Naturais, Universidade Federal do Espírito Santo. Av. Fernando Ferraci, 524, Campus Universitário de Goiabeiras, 29075-910, Vitória, ES, Brasil. Correspondência para/Correspondece to: S.R.F. ENUMO. E-mails: <soniaenumo@terra.com.br>, <enumosrf@npd.ufes.br>.

2 Doutoranda, Bolsista CAPES, Programa de Pós-Graduação em Psicologia, Centro de Ciências Humanas e Naturais, Universidade Federal do Espírito Santo. Vitória, ES, Brasil.

3 Professora Doutora, Centro Universitário Vila Velha. Vila Velha, ES, Brasil.

Apoio: CNPq, FACITEC-PMV, CAPES e Programa de Pós-Graduação em Psicologia, Universidade Federal do Espírito Santo. Agradecimentos à Professora Doutora Tatiane L. Dias (UNEMAT), à mestranda Flávia A. Turini e à bolsista de Iniciação Científica/CNPq Alyni P. Gottardi. 
Tradicionalmente, as crianças com dificuldades de aprendizagem têm sido estudadas em relação à memória, pensamento, raciocínio, percepção, linguagem e comportamento, resume McKinney (1989). Entretanto, Oatley e Nundy (2000) consideram o componente afetivo o determinante primário do desempenho na escola, o qual estaria relacionado às emoções, às atitudes e aos interesses.

Segundo Rutter (1989), desde 1920 já foram encontrados padrões de comorbidade entre saúde física fraca e problemas psicológicos ou educacionais na Inglaterra. No primeiro estudo epidemiológico em 1958, identificou-se alta freqüência de problemas emocionais e comportamentais em crianças entre seis e doze anos, como sintomas de desatenção, hiperatividade, depressão e comportamentos desviantes, associados a problemas emocionais e à leitura. Na revisão da área, esse autor indicou um papel causal concomitante para as adversidades familiares, disfunções orgânicas do sistema nervoso e dificuldades de temperamento da criança. Queixas recorrentes de dor abdominal foram comuns entre as crianças, estando freqüentemente associadas a problemas psicológicos, assim como dor de cabeça e de estômago, associadas à depressão materna e a problemas na relação conjugal. O ambiente escolar esteve fortemente associado às características do êxito escolar da criança, sendo mais predominante do que o efeito familiar nas habilidades de leitura aos sete anos. A freqüência às aulas é tida como uma boa medida afetiva e comportamental, indicando se a criança evita ou gosta da escola (Oatley \& Nundy, 2000).

Em nosso país, Carvalho, Linhares e Martinez (2001) encontraram uma maior incidência de bronquite alérgica, desmaios, otite crônica, pneumonia, queixas relatadas de asma, crise respiratória e enurese noturna, entre outras, em 20 crianças nascidas com muito baixo peso( $\leq 1500 \mathrm{~g})$, com idade entre oito e dez anos, que também apresentavam maior freqüência de repetência escolar e alguns indicadores de problemas de comportamentos, como recusa escolar, agitação e impaciência, do que seus pares nascidos a termo, esses também com queixas de dificuldade de aprendizagem. No geral, contudo, os dois grupos não apresentaram diferenças significativas quanto a algumas variáveis importantes, como função cognitiva (normal), desempenho escolar (baixo), condições atuais de saúde (doenças pulmonares e problemas respiratórios) e dificuldade de aprendizagem.

Graminha (1994) conduziu um dos poucos estudos epidemiológicos da área, com 1731 escolares da pré-escola à 6a série, em Ribeirão Preto, SP. Dentre 36 tipos de problemas comportamentais estudados, mais da metade dos pais se referiu ao filho como desobediente, mal-humorado e nervoso, muito agarrado à mãe; entre $40 \%$ e $50 \%$ dos pais citaram problemas como dor de cabeça, dificuldade de alimentação, de sono, medo, agitação, impaciência e timidez.

Essas questões se tornam relevantes, como mostram as pesquisas que associam a depressão e as disfunções sociais e comportamentais às dificuldades na família, na escola e nas relações com colegas e amigos (Santos \& Marturano, 1999). Andriola e Cavalcante (1999) consideram que, para caracterizar uma depressão infantil, pelo menos quatro desses sintomas devem estar presentes por duas semanas antes da avaliação: humor disfórico, autodepreciação, agressividade ou irritação, distúrbios do sono, queda do desempenho escolar, diminuição da socialização, modificação de atitudes em relação à escola, e perda da energia habitual, do apetite e/ou peso. Esses autores encontraram uma prevalência de 3,9\% de depressão, sem diferenças significativas entre os sexos, entre 345 alunos da pré-escola (5,6 anos, em média) de Fortaleza, CE, avaliados pela Escala de Sintomatologia Depressiva para Mestres (ESDM); esses dados se encontram dentro da faixa encontrada pela literatura da área (3,0\% a 6,0\%).

Quanto à relação entre stress e escola, foram feitos estudos em nosso país, como o realizado por Tanganelli e Lipp (1998) em 158 alunos de 1a a 4a série, com e sem dificuldades escolares, utilizando o Inventário de Sintomas de Stress Infantil (ISSI). Observou-se alta freqüência de sintomas de stress ( $55 \%$ da amostra), em particular entre os alunos mais fracos (67\%), com maior freqüência entre as meninas, predominando os sintomas cognitivos (ficar preocupado com coisas ruins que podem acontecer, ter medo, vontade de chorar). Os alunos classificados como fracos mostraram ter dificuldade para prestar atenção e sentiam-se tristes.

Os sintomas somáticos de stress mais comuns foram: mãos suadas, dor de barriga, não conseguir ficar parado e quieto num lugar por muito tempo e coração 
acelerado. Segundo Lipp, Souza, Romano e Covolan (1991), o stress infantil ainda é demonstrado no nível físico por sintomas como dor de cabeça, náuseas, tique nervoso, ranger de dentes, e enurese noturna; e no nível psicológico por agressividade e pesadelos.

Em relação próxima com os quadros de stress está a ansiedade. Segundo Rutter (1989), os eventos estressantes e o baixo status social apareceram como relacionados à ansiedade, variável pouco estudada em nível epidemiológico. Multifatores estão associados à propensão à ansiedade, incluindo-se uma dimensão cognitivo-social e outra ligada ao contexto biológico (tensão nervosa e stress), segundo dados da literatura resumidos por Nascimento (2001). Essa autora, enfatizando a dimensão interpessoal da ansiedade, mostrou haver relações entre o nível de ansiedade de alunos da $4^{a}$ e da 8 a série, medido pelo Inventário de Ansiedade Traço-Estado para Crianças (IDATE-C), e o nível de ansiedade de seus pais (IDATE).

No geral, esses estudos indicam a necessidade de intervenções e medidas preventivas, atuando em todos os níveis direta ou indiretamente envolvidos com o desempenho acadêmico, ou seja, a família e o ambiente escolar, além da própria criança, como também propõem outros autores a exemplo de Shapiro e Bradley (1999) e Trapani e Gettinger (1999). Nesse sentido, com base nos dados da literatura expostos, a presente pesquisa pretendeu identificar, descrever e analisar possíveis relações entre emoções relatadas e saúde física de alunos com dificuldades de aprendizagem, cursando séries iniciais do ensino fundamental, comparando-os com alunos sem dificuldade de aprendizagem. Partiu-se do pressuposto de que os alunos com dificuldade de aprendizagem apresentariam mais indicadores de ansiedade, de depressão e de stress, mais doenças físicas diagnosticadas, menor peso e menor altura em relação aos colegas da mesma idade, maior absentismo por motivos de saúde, e mais queixas físicas do que seus colegas sem dificuldades de aprendizagem.

\section{Método}

\section{Participantes}

Participaram da pesquisa 60 alunos com idade entre 8 e 14 anos ( $M d=9$ anos), sendo 31 meninos e 29 meninas, cursando, em 2002, a 3a e a 4a série do ensino fundamental em 6 classes de uma escola pública de Vitória, ES. Os participantes foram avaliados quanto ao desempenho acadêmico pelo Teste de Desempenho Escolar (TDE) (Stein, 1994) no final de 2001, quando estavam na $2^{\text {a }}$ e $3^{\text {a }}$ série escolar. Numa etapa inicial, de acordo com os resultados no TDE aplicado a 144 alunos cursando a $2^{a}$ e $3^{a}$ série dessa escola em 2001, foram formados dois grupos descritos a seguir:

- Grupo 1 (G1): formado por 30 alunos classificados no nível inferior do TDE (média= 75 pontos), considerados como tendo dificuldade de aprendizagem (DA). A maioria desses alunos do G1 tinha entre 8 e 9 anos (20 alunos) e cursava a 3a série (18) em 2002; as mães e os pais desses alunos tinham ensino fundamental incompleto e exerciam profissões não qualificadas, segundo classificação de Soares e Fernandes (1989).

- Grupo 2 (G2): formado por 30 alunos classificados pelo TDE nos níveis médio e superior (média = 107,1 pontos; foi necessário incluir os dois níveis, pois o número de alunos classificados no nível inferior era muito maior: $73 \%$ do total de 144 alunos dessas séries), considerados sem dificuldade de aprendizagem (sem DA). Havia mais meninas (19); a faixa etária dos alunos variou entre 8 e 11 anos (maioria entre 8 e 9 anos e na $2^{\text {a }}$ série); tinham pais e mães com ensino fundamental incompleto, trabalhando em profissões não qualificadas. Os responsáveis assinaram o termo de compromisso para participar da pesquisa, que foi autorizada pelo Comitê de Ética do Programa de Pós-Graduação em Psicologia.

Na segunda etapa da pesquisa, participaram somente 14 desses alunos (23,3\% da amostra inicial), sendo 7 alunos da 3a série e 7 alunos da 4a série, sendo 6 meninas e 8 meninos, na faixa etária entre 8 e 11 anos ( $M d=9$ anos), separados em dois subgrupos: 1) grupo 1 a (G1a): com dificuldade de aprendizagem (DA) e com indicações de risco para problemas emocionais e/ou de saúde, sendo 5 meninos e 2 meninas, na faixa etária entre 8 e 11 anos ( $M d=9$ anos); um aluno tinha uma defasagem de um ano entre a idade e a série; 2) grupo 2a (G2a): sem dificuldade de aprendizagem (sem DA) e com indicações de risco para problemas emocionais e/ou de saúde, sendo 3 meninos e 4 meninas, na faixa etária entre 8 e 10 anos ( $\mathrm{Md}=8$ anos); havia 2 alunos adiantados na idade em relação à série. 


\section{Procedimento}

A pesquisa foi realizada em duas etapas, descritas a seguir:

Etapa 1: em novembro de 2001, selecionou-se a amostra inicial, posteriormente dividida em dois grupos a partir da aplicação e resultados no Teste de Desempenho Escolar (TDE) (Stein, 1994), havendo o auxílio de bolsistas de iniciação científica. O TDE é um teste brasileiro, composto por três subtestes: Leitura, Escrita e Aritmética, que classifica os resultados em cada série escolar em: inferior, médio e superior. Para as séries avaliadas, tem-se a seguinte classificação para a pontuação de acertos, segundo as normas do TDE: $2^{2}$ série - inferior: $\leq 86$; médio: 87-105; superior: $\leq 106$; e 3a série-inferior: $\leq 101$; médio: 102-112; superior: $\leq 113$.

Para a identificação dos alunos com mais indicadores de problemas emocionais e de saúde, foram aplicados, em maio e junho de 2002, quatro instrumentos descritos a seguir:

1) Inventário de Ansiedade Traço-Estado para Crianças (IDATE-C) (Biaggio, 1983): avalia a ansiedade em crianças. Foi aplicado nos 60 alunos em grupo, por classe escolar, por uma pesquisadora, que também auxiliou no preenchimento, quando necessário;

2) Escala de Stress Infantil (ESI) (Lipp \& Lucarelli, 1998): aplicada seguindo o mesmo procedimento do item anterior, objetiva identificar a existência ou não de stress;

3) Escala de Sintomatologia Depressiva para Mestres (ESDM) (Dias, Barbosa, Gaião \& Di Lorenzo, 1997): seis professoras dos alunos da amostra responderam a essa escala, em entrevista individual, com normas nacionais;

4) Questionário com 13 questões sobre doenças e queixas somáticas (dor de cabeça, de barriga, olhos, ouvidos) dos alunos e outras informações relevantes, como o estado geral do aluno durante a aula (sonolência, abatimento e lentidão, por exemplo): essas seis professoras responderam, também individualmente, a questões classificadas numa escala de três pontos (quase nunca, algumas vezes, sempre), além das faltas por motivos de saúde e uma estimativa de peso e altura de

142 cada um dos alunos em relação aos colegas. Esse questionário foi também preenchido pela pesquisadora durante a entrevista.

Os instrumentos foram corrigidos de acordo com as normas especificadas nos manuais, acrescentando-se algumas categorias classificatórias para melhor enquadrar os resultados, mantendo-se, porém, os critérios propostos pelos autores.

Etapa 2: A partir do processamento inicial dos dados das escalas de stress, ansiedade e depressão, e do questionário para professores, foi composta uma nova amostra de 14 alunos com indicações de risco para problemas emocionais e/ou de saúde, segundo os critérios: ter indicação em duas ou mais escalas; ter indicação pelas professoras quanto a doenças; queixas e sintomas físicos; como dor de cabeça; lentidão e abatimento; ter muitas faltas por motivos de saúde.

Esse grupo de alunos que estavam sujeitos a agravamento dos quadros físico e emocional foi dividido novamente em dois subgrupos, segundo a classificação inferior e superior no TDE. Esses 14 alunos selecionados foram entrevistados individualmente, com gravação em áudio, seguindo um protocolo de entrevista para alunos, contendo: (a) 22 perguntas a respeito da rotina em casa quanto a sono, lazer e execução de tarefas escolares; da rotina na escola (tempo estimado de execução das atividades em classe), situações e conteúdo de disciplinas consideradas as mais agradáveis e as mais rejeitadas na escola; da interação com companheiros; dos motivos de faltas escolares; e dos comportamentos moralmente inadequados (pegar coisas de outra pessoa); (b) 46 itens a serem marcados pelo pesquisador de acordo com o relato do aluno sobre a presença ou ausência de queixas de dor (7 tipos), de sintomas físicos (21 tipos), reações emocionais (6 tipos) e respostas comportamentais (12 tipos).

Foram explorados os eventos antecedentes e conseqüentes das respostas dadas pelos alunos e realizada análise funcional, seguindo o modelo adaptado de Johnson, Baumgart, Helmstetter e Curry (1996), com a comparação dos dados obtidos dos dois subgrupos. Registrou-se também o número de faltas cometidas individualmente pelos alunos nos dois bimestres anteriores, obtido na secretaria da escola (março a junho de 2002).

Os dados foram submetidos à análise estatística paramétrica e não-paramétrica, adotando-se o nível de 
significância de $5 \%(p \leq 0,05)$, comparando os dois grupos (G1 e G2) em termos de resultados brutos e classificatórios. Além disso, foi feita uma análise estatística (média, desvio-padrão, amplitude de variação e percentis), separada por sexo, para saber se a amostra dos 60 alunos tinha o mesmo perfil de desempenho da amostra original da escala de ansiedade (IDATE-C). Os dados ficaram dentro do desvio-padrão, sendo, então, utilizadas as normas originais.

\section{Resultados}

Dos 60 alunos da amostra inicial, 21,6\% tiveram indicações na escala ESI de stress, sendo 6 alunos (20\%) do G1 e 7 do G2 (23,3\%); 31,6\% dos alunos (19) tiveram indicações em pelo menos em uma das escalas de ansiedade IDATE-C (G1:20\%; G2:43,3\%) e 1 aluno (1,6\%; do G1) teve indicação para a escala de depressão ESDM. Apesar das diferenças encontradas entre os dois grupos, a análise de variância não paramétrica - utilizada para dados que não seguem uma distribuição normal (não categóricos), feita através do teste Kruskal-Wallis, utilizado para duas ou mais amostras (Fonseca \& Martins, 1994; Gomes, 1981) - não encontrou diferenças significativas entre os dois grupos de alunos quanto aos resultados nas escalas de stress (ESI), ansiedade (IDATE-C) e depressão (ESDM). O mesmo ocorreu quanto aos indicadores de doenças físicas e faltas por motivo de doença fornecidos pelas professoras.

Como esses dados pareciam discrepantes das observações feitas pelas aplicadoras e entrevistadoras, foi utilizado um teste paramétrico (Anova) para cálculo de variação entre os dois grupos de alunos, baseando-se agora nos resultados brutos e não na classificação dos instrumentos psicométricos, obtendo-se uma diferença significativa apenas na escala de depressão (ESDM) para G1, que teve uma média de 5,56 pontos brutos nessa escala, contra a média de 3,46 pontos do G2.

Assim, na medida em que os grupos se apresentaram como homogêneos nos itens avaliados, foram utilizados todos os 60 alunos para a análise de correlação não paramétrica entre os resultados nas escalas e indicadores da pesquisa, feita com o coeficiente de Spearman. Nos instrumentos psicométricos, as únicas correlações encontradas referem-se aos resultados internos da escala de ansiedade (IDATE-C): houve correlação significativa a $1 \%$ de probabilidade entre o resultado geral e das subescalas (de 0,785 com a ansiedade-traço e 0,658 com a ansiedade-estado), mas não nas subescalas entre si (índice de 0,112 de correlação).

Nos demais dados, para toda a amostra de 60 alunos, ter indicações para doenças físicas correlacionou-se significativamente a $1 \%$ de probabilidade com faltas por motivo de doença $(0,473)$ e sonolência e baixa energia em sala de aula $(0,454)$, e vice-versa. Faltas por motivos de doenças correlacionaram-se com queixas de dor $(0,556)$ e sonolência e baixa energia em sala de aula $(0,339)$, e vice-versa. Houve também correlação a 5\% de probabilidade entre doenças físicas e peso/altura discrepante dos colegas $(0,267)$.

Fazendo a mesma análise para cada grupo, no G1, encontraram-se correlações para os 30 alunos entre: (a) a escala geral de ansiedade e suas subescalas (IDATE-C), semelhante à amostra geral; (b) as subescalas de ansiedade entre si (IDATE-C); fato que não ocorreu na amostra geral e no G2; (c) ter indicações de doença física com faltas por motivo de doença e com queixas de dor; (d) faltas por motivo de doença com a subescala de ansiedade-estado da IDATE-C, com ocorrência de doença física, queixas de dor, e com sonolência/apatia. Foi o subgrupo com o maior número de correlações entre as variáveis (Tabela 1).

No G2 (sem DA), observou-se o mesmo padrão da amostra geral, encontrando-se correlações entre: (a) resultado geral na escala de ansiedade e suas subescalas (IDATE-C), semelhante à amostra geral; (b) a subescala de ansiedade-traço (IDATE-C) e ter ocorrência de doença física; (c) ter indicações de doença física com faltas por motivo de doença, e com sonolência/baixa energia em sala de aula; (d) faltas por motivo de doença com queixas de dor (Tabela 1).

\section{Subamostra}

\section{Alunos com indicações de problemas emocionais e de saúde física}

Os dois subgrupos, totalizando 14 alunos selecionados por terem indicações de problemas de saúde física e/ou emocionais segundo os critérios já 
Tabela 1. Correlações entre indicações de stress (S), ansiedade-estado (AE), ansiedade-traço (AT), nas duas escalas de ansiedade (A), depressão (D) e ocorrência de doença física (F1), faltas escolares por doença (F2), peso/altura discrepante da média de idade (F3), queixas de dor (F4) e sonolência/apatia (F5), a partir da aplicação da ESI, IDATE-C, ESDM e do questionário de saúde física, nos alunos com (G1) e sem (G2) dificuldade de aprendizagem (DA) $(n=60)$.

\begin{tabular}{|c|c|c|c|c|c|c|c|c|c|c|}
\hline & s & $A E$ & AT & A & D & F1 & F2 & F3 & $\mathrm{F} 4$ & F5 \\
\hline \multicolumn{11}{|c|}{ G1- DA $(n=30)$} \\
\hline$S$ & 1 & 081 &,- 195 &,- 045 &, 339 &,- 166 &,- 121 &,- 188 &,- 056 &,- 155 \\
\hline $\mathrm{AE}$ & & 1 & $423^{*}$ &, $779 * *$ &,- 073 &,- 131 &, $385^{*}$ &,- 277 & 280 &,- 170 \\
\hline AT & & & 1 & $844^{* *}$ &,- 073 &,- 131 &, 171 & ,139 & 280 &,- 170 \\
\hline$A$ & & & & 1 &,- 092 &,- 165 & 241 & 000 & 293 &,- 276 \\
\hline D & & & & & 1 &,- 062 &,- 122 & 263 &,- 152 & 199 \\
\hline F1 & & & & & & 1 &, $509^{* *}$ &, 236 &, $408^{*}$ &, 356 \\
\hline F2 & & & & & & & 1 &,- 154 &, $653^{* *}$ &, $408^{*}$ \\
\hline F3 & & & & & & & & 1 &, 000 &,- 094 \\
\hline F4 & & & & & & & & & 1 & ,191 \\
\hline F5 & & & & & & & & & & 1 \\
\hline
\end{tabular}

\begin{tabular}{|c|c|c|c|c|c|c|c|c|c|c|}
\hline \multicolumn{11}{|c|}{ G2- sem DA $(n=30)$} \\
\hline S & 1 &, 225 &,- 085 &, 096 & - &,- 183 &,- 246 &, 012 & ,278 &,- 124 \\
\hline$A E$ & & 1 &,- 113 &, $516^{* *}$ & - &,- 201 &,- 067 &,- 193 &,- 154 &,- 154 \\
\hline AT & & & 1 &, $774^{* *}$ & - & $389^{*}$ &, 224 & 264 &, 118 &, 118 \\
\hline A & & & & 1 & - &, 237 &, 179 & ,136 &, 025 & 025 \\
\hline D & - & - & - & - & - & & & - & - & - \\
\hline $\mathrm{F} 1$ & & & & & - & 1 &, $447^{*}$ & ,302 &, 079 &, $604^{* *}$ \\
\hline F2 & & & & & - & & 1 &, 135 &, $388^{*}$ &, 176 \\
\hline F3 & & & & & - & & & 1 &,- 154 & ,202 \\
\hline F4 & & & & & - & & & & 1 &,- 118 \\
\hline F5 & & & & & - & & & & & 1 \\
\hline
\end{tabular}

${ }^{*} p \leq 0,05=$ significativo $5 \%$ de probabilidade; ${ }^{*} \leq 0,01=$ significativo a $1 \%$ de probabilidade; coeficiente de Spearman.

descritos no procedimento, foram comparados entre si pelo teste não paramétrico Kruskal-Wallis, e não se encontraram diferenças significativas em todos os itens avaliados; ou seja, essa segunda amostra também era homogênea. Por esse motivo, todos os 14 alunos foram computados nos cálculos de correlação feitos para os resultados dos instrumentos e indicadores obtidos com as professoras e o número de faltas escolares.

Essa análise dos dados dos 14 alunos apontou correlações significativas, como entre o resultado geral da escala de ansiedade (IDATE-C) e a subescala de ansiedade-traço - 0,846 a 1\% de probabilidade (e não com a ansiedade-estado, como ocorreu com a amostra : geral) e entre faltas por doença e queixas de dor 罗 (correlação significativa de 0,559 a 5\% de probabilidade).

Apesar de os dois grupos serem semelhantes estatisticamente quanto aos itens avaliados pelos instrumentos, os dados brutos e individualizados apresentaram diferenças que foram analisadas. Os alunos do G1a tiveram uma maior média de faltas no semestre (7,2 faltas/aluno; amplitude de variação - $A V=0-17)$ que os alunos do G2a (2,6 faltas/aluno; $A V=0-8) ; 3$ alunos (0,43 do subgrupo) tiveram indicação de stress (G2a: um aluno); (c) 1 aluno teve indicação de 3 tipos de stress: psicofisiológico, psicológico e psicológico com componente depressivo, o que não ocorreu no G2a; uma média maior de indicadores de depressão (6,71/aluno; $A V=1-20$ pontos) em relação ao G2a (4,57/aluno; $A V=0-10$ pontos); um aluno com clara indicação de depressão, segundo critério de corte da escala (16 pontos); o que não ocorreu no G2a; e um aluno com indicação de ansiedade geral (estado e traço) no IDATE-C; fato não observado no G2a.

Os alunos do G2a (sem DA) tiveram resultados maiores do que G1a na escala de ansiedade (G2a: cinco alunos; G1a: dois), e na indicação de ansiedade-traço (G2a: três alunos; G1a: um aluno). Os dois grupos de alunos tiveram resultados semelhantes somente na subescala de ansiedade-estado (dois alunos cada grupo) 
da IDATE-C, possivelmente relacionados ao contexto de estarem sob avaliação.

Além das diferenças entre os subgrupos nos resultados das escalas aplicadas, observou-se que as professoras relataram perceber mais sintomas somáticos nos alunos do G1a (2,28/aluno) do que nos alunos do G2a (1,57/aluno). Esses dados são coerentes com as queixas apresentadas pelos alunos dos dois grupos nas entrevistas. Apresentar-se abatido e sem energia na sala de aula foi comum aos dois subgrupos de alunos; mas queixas de dor de cabeça e na barriga e sonolência só foram percebidas pelas professoras em relação ao G1a. Queixas de enjôo só foram relatadas pelas professoras em relação aos alunos sem DA.

Houve discrepâncias entre o número real de faltas às aulas por parte dos alunos e o número relatado pelas professoras, mas manteve-se a diferença na proporção: mais faltas no G1a (até 5 faltas, havendo aluno com até 17 faltas, que correspondem a 20\% das aulas do semestre) do que no G2a (até 2 faltas, segundo as professoras; mas, segundo os registros, faltaram até 8 vezes). Em resumo, as professoras deram indicações de que percebem os alunos com DA menos assíduos do que os demais. Em relação à avaliação das professoras sobre a adequação do desenvolvimento físico (peso e altura) dos alunos em questão, quando comparados com os demais de sua turma, observa-se que a maioria (5) dos alunos com DA (G1a) estava na faixa esperada para sua idade; já os alunos do G2a formavam um grupo mais heterogêneo.

\section{Entrevistas com os alunos}

A partir da entrevista com os 14 alunos, observou-se que houve uma freqüência maior de queixas físicas de dor de cabeça e reação emocional de medo, seguidas de sintomas físicos de alergia; e depois, por respostas comportamentais, como ter dificuldades para dormir, morder blusa, lápis ou unhas, junto com reações emocionais, como ter a sensação de não servir para nada. Houve menor freqüência de sintomas físicos de azia, vômitos, dor nos olhos e ouvido, e reações emocionais, como ranger os dentes. No geral, os alunos do G1a apresentaram uma freqüência maior de: (a) sintomas físicos (8,3 queixas/aluno), como, por exemplo, muito cansaço, gripe, sudorese, diarréia, do que os do
G2a (7,1 queixas/aluno); (b) dores de cabeça, de garganta e tosse (G1a: 3,4/aluno; G2a: 2,7/aluno); (c) respostas comportamentais, como morder gola/lápis, roer unhas, ficar mexendo as mãos, não conseguir ficar quieto no lugar (G1a:5/aluno; G2a:4,7/aluno).

Os alunos do G2a (sem DA) apresentaram pequena diferença em relação ao G1a quanto aos relatos de reações emocionais (3,1/aluno), como ter sensação de não servir para nada/ser inútil, sentir muita tristeza. Alunos do G1a relataram ter mais vontade de chorar, com ou sem motivo. A diferença entre os subgrupos foi apenas um ponto a mais para G2a.

\section{Análise funcional dos relatos}

De modo geral, não foram identificadas diferenças entre G1a e G2a em relação aos eventos antecedentes para comportamentos e efeitos colaterais da emoção.

\section{Eventos relevantes relacionados à interação familiar e ao ambiente social}

Os alunos relataram sentimentos de muita tristeza, vontade de morrer, de ficar quieto, e de chorar com ou sem motivo, de forma contingente a contextos de conflitos familiares, incluindo-se episódios de briga entre os pais. Os medos relatados parecem contingentes a situações relacionadas a dificuldades para dormir, a episódios de morte na família ou vizinhança, ou contato com eventos de violência urbana (assalto, ladrão). Apesar da alta freqüência de relatos de medo, todos parecem comuns à infância e ao contexto social em que vivem (moradia na base de um morro, perto de favela, onde ocorrem brigas de bar principalmente). Os alunos do G1a relataram com maior freqüência acordar durante a noite e chamar a mãe, comportamentos esses que estariam associados a pesadelos.

Com base nos dados da entrevista, foram organizadas as informações fornecidas pelos alunos em relação à rotina familiar, observando-se, agora, diferenças entre os alunos do G1a e G2a. Foi identificada a incidência de eventos familiares relacionados ao cumprimento de obrigações escolares e descanso. No geral, os alunos do G2a (sem DA) relataram melhor organização familiar, facilitadora do desempenho escolar, 
como ter rotinas que envolvem seu encaminhamento diário à escola, a especificação de contingências para a realização de tarefas escolares em casa; sono noturno igual ou superior a oito horas, em horário adequado; realização do dever de casa em horário adequado (durante o dia, após o descanso); ajuda de membros da família na realização do dever de casa; e menor tempo despendido na realização dos deveres escolares. Esses dados em relação ao G1a mostram uma discrepância em todos os itens avaliados, exceto no relacionado à ajuda no dever de casa. A quantidade de sono era semelhante; entretanto, dois alunos do G1a relataram ter horários de sono inapropriados (dormiam muito tarde e acordavam tarde).

\section{Eventos relacionados ao ambiente escolar}

O sentimento de inutilidade parece relacionado a críticas dos professores (e da mãe) e à nota baixa. No geral, não foram observadas diferenças entre os relatos de preferências dos alunos na escola, no que se refere ao comportamento dos professores e conteúdos de disciplinas, por exemplo. Também disseram gostar mais das atividades propostas pela escola; havendo somente um aluno que relatou vontade de ir embora ou fugir da escola contingente a alguma interação social malsucedida na escola, com professor (críticas) ou com colegas (implicar, brigar).

Na situação de sala de aula, os alunos disseram sentir tédio às vezes, ficando então com dificuldades em se manter na atividade ou ficar quieto no lugar. Alguns relataram se levantar, outros disseram arrumar desculpas para sair da classe, ou disseram se engajar em atividades de rabiscar e desenhar. Os alunos do G1a avaliaram com mais freqüência as atividades escolares como difíceis. Esses alunos se percebiam como mais lentos na realização das atividades em sala de aula, e pareciam adiar a execução do dever de casa. Esses contextos apareceram relacionados à dor de cabeça e a sentimentos de confusão.

Nos relatos dos alunos de ambos os grupos, o comportamento do professor pode ser considerado fator interveniente na preferência por conteúdos escolares. Professores que organizavam atividades lúdicas (jogos) e que apresentavam comportamentos empáticos eram preferidos àqueles que faziam críticas, gritavam e ameaçavam punir a indisciplina. Os episódios de briga na escola foram citados por alunos de ambos os grupos com alta freqüência, especialmente na situação de recreio, momento em que não existia qualquer atividade monitorada por adultos.

Diante de uma situação de briga, houve alunos, em ambos os grupos, que relataram sempre se envolverem, enquanto outros sempre evitavam o envolvimento nesses episódios. Esses últimos pareciam ter mais dificuldades para manter vínculos de amizade e, de modo geral, avaliavam negativamente os colegas ("não são amigos"; "são uns traíra"). As faltas escolares foram justificadas pelos alunos por motivo de saúde, preguiça, ou ausência/quebra de rotina familiar.

\section{Discussão}

Os dados dos 60 alunos aqui estudados contrariaram aqueles obtidos em pesquisas feitas na área sobre stress, também utilizando a Escala de Stress Infantil, com alunos do ensino fundamental, como o estudo de Tanganelli e Lipp (1998), que encontrou uma alta freqüência de sintomas de stress em alunos classificados como "fracos" no desempenho escolar, em contraposição ao grupo de alunos classificados como "fortes". Destoam também esses dados daqueles encontrados por Medeiros, Loureiro, Linhares e Marturano (2000). Também nesse aspecto de saúde, os dados da presente pesquisa não confirmaram haver diferenças significativas entre alunos com e sem dificuldades na escola.

A comorbidade, encontrada por Rutter (1989), entre saúde física fraca e problemas educacionais, com alta freqüência de queixas somáticas (dor de cabeça, dores abdominais, por exemplo), em crianças dessa faixa etária, não foi identificada na presente pesquisa. Da mesma forma, os dois grupos de alunos desta pesquisa não se diferenciaram significativamente quanto aos indicadores de ansiedade, diferentemente das indicações de trabalhos epidemiológicos e de revisão da literatura da área, realizados por Rutter (1989) e McKinney (1989).

Nem mesmo a freqüência às aulas, considerada um forte indicador de adesão ou não à escola, segundo Oatley e Nundy (2000), mostrou diferenças significativas entre os alunos com e sem DA. Fatores como maior acompanhamento familiar e/ou maior controle por 
parte da escola podem estar relacionados a esses resultados, como indicam Magna e Marturano (1998), Marturano (1997, 1998) e Marturano e Ferreira (2004), apoiadas em dados de pesquisas nacionais e internacionais sobre as variáveis intra-escolares, extra-escolares e familiares relacionadas ao desempenho escolar.

Somente em relação ao maior número de meninos no grupo com DA, coerentemente com dados epidemiológicos de Rutter (1989), e aos indicadores de depressão, houve concordância entre os dados desta pesquisa e das demais citadas anteriormente. Lembramos, porém, que a diferença significativa foi encontrada apenas quando foram utilizados os dados brutos e não a classificação geral de depressão proposta originalmente pela escala utilizada (ESDM). A incidência de um aluno (1,6\% da amostra total) indicado com sinais de depressão se encontrou abaixo da incidência de 3,0\% a 6,0\%, citada na literatura internacional e nacional (Andriola \& Cavalcanti, 1999).

Diante de tantos dados esperados e não confirmados, e afastando a hipótese de inadequação dos instrumentos aos objetivos desta pesquisa, resta buscar algumas possíveis explicações, considerando, com Meltzoff (2001, p.112), que a "... busca por um significado psicológico dos resultados significativos não pode ser guiada exclusivamente pelos valores de $p^{\prime \prime}$, como se discute a seguir:

1) esses alunos pesquisados, como grupo, poderiam não ter problemas emocionais, comportamentais e físicos associados a desempenho escolar devido a variáveis ecológicas, ou seja, relacionadas a algum fator local, como a escola que freqüentam, o bairro ou a cidade em que vivem (Rutter, 1981), o que parece pouco provável frente aos indicadores educacionais e de saúde pouco promissores do ES e sua capital (Instituto Brasileiro de Geografia e Estatística - IBGE, 2002);

2) os instrumentos psicométricos utilizados poderiam não ser adequados em termos de sensibilidade para detecção dos problemas a que se propõem, contradizendo todo trabalho de elaboração ou padronização indicado nos manuais;

3) as normas desses instrumentos poderiam não ser adequadas para a amostra estudada; fato que não ocorreu pelo menos em relação à escala de ansiedade (IDATE-C), pois, como mostraram os dados estatísticos, as respostas dessas crianças estavam dentro do desvio-padrão especificado no manual. Nesse ponto, convém destacar que os dados da escala em questão foram os únicos a apresentarem índices de correlação interna (as subescalas de ansiedade-estado e de ansiedade-traço se correlacionaram com os dados da escala geral de ansiedade, mostrando coerência interna entre as escalas do IDATE-C);

4) a adoção de um critério de classificação objetivo e externo à escola, no caso o Teste de Desempenho Escolar (TDE), para identificar os casos de dificuldade de aprendizagem poderia ter diferenciado a amostra em relação às amostras dos outros estudos utilizados como comparação (Andriola \& Cavalcanti, 1999; McKinney, 1989; Medeiros et al., 2000; Rutter, 1989; Tanganelli \& Lipp, 1998). Esses estudos, em geral, se basearam em indicações informais da família e da escola, especialmente na indicação de professores, ou trabalharam com amostras clínicas, que foram encaminhadas a serviços especializados por apresentarem queixas de dificuldade de aprendizagem.

Parece não ser uma única hipótese que explicará as discrepâncias de dados encontradas nesta pesquisa; mas alguns dados sugerem uma explicação relacionada às características dos instrumentos utilizados, pois os dados obtidos junto às professoras por questionário e em entrevista com os alunos estão coerentes com as previsões da literatura quanto à relação entre doença física ou queixas somáticas e baixa energia em sala e faltas à escola entre todos os alunos, e entre discrepâncias de peso/altura em relação aos colegas e doença física.

Cruzando as informações obtidas com esses outros instrumentos - questionários e entrevistas - com as escalas e de acordo com as correlações observadas, conclui-se que as relações entre doença física e variáveis emocionais foram confirmadas para o conjunto da amostra de 60 crianças entre 8 e 14 anos de idade, por meio de dados obtidos por entrevistas com professores. Metodologicamente, chama a atenção a falta de correlação entre os dados de stress e ansiedade, pois há vários itens semelhantes entre essas escalas, respondidas de forma quase semelhante.

Apesar de os dados obtidos por questionário com as professoras terem tido mais correlação com indicadores de depressão e problemas físicos nos alunos 
do que os resultados nas escalas de stress e ansiedade, considerou-se importante o uso desses últimos de forma complementar na identificação de alunos com mais indicadores de problemas emocionais e físicos durante a constituição da segunda amostra analisada nesta pesquisa.

Considerando agora o subgrupo de 14 alunos com maior risco de problemas emocionais e/ou físicos, também não se encontraram diferenças significativas estatisticamente. Entretanto, a mesma postura de não considerar apenas os dados quantitativos e sua significância estatística, como sugere Meltzoff (2001), levou à realização de entrevistas, procurando obter dados que possibilitassem uma análise funcional das queixas identificadas nos instrumentos anteriores, juntamente com novos dados fornecidos pelos alunos. Só então as diferenças entre os dois subgrupos começaram a aparecer, inclusive quantitativamente.

Como previam dados da literatura (Rutter, 1989), os professores não perceberam tão facilmente problemas quando comparadas suas informações com as dadas pelos alunos; em outros termos, a coleta de dados feita diretamente com o sujeito é mais informativa do que quando feita por fontes indiretas.

Esses alunos com DA, quando comparados com o subgrupo sem problemas escolares, relataram mais doenças e sintomas físicos, queixas de dor de cabeça, principalmente, e comportamentos indicativos de stress, ansiedade e depressão, com maior freqüência de medos e pesadelos. Os problemas de saúde (relacionados ao aparelho respiratório, como asma, principalmente) confirmam os dados de Carvalho et al. (2001) e parecem ter relações com quadros de ansiedade, sendo precedidos por reações emocionais como raiva e tristeza. Esse grupo, entretanto, não se diferencia daquele sem DA quanto à funcionalidade dos comportamentos apresentados, como afirma Marturano (1997); parece, sim, existir um maior acúmulo de eventos estressantes : para os alunos com dificuldades na escola.

Foi na rotina familiar, porém, que ocorreram mais diferenças entre os dois subgrupos. Os alunos sem DA relataram ter uma rotina de encaminhamento à escola mais sistemática, com contingências claras para realização dos deveres escolares em casa, horário de sono mais saudável (não deitar depois da meia-noite e acordar tarde no dia seguinte, como ocorreu no grupo com DA), e realização das tarefas com maior rapidez.

Foram identificadas, assim, pela entrevista, variáveis relacionadas à família que parecem ter influências no desempenho acadêmico, assim como variáveis do contexto escolar em termos de ambiente físico e social, com destaque para a relação professor-aluno, além das variáveis da própria criança, que afetavam 23,3\% dessa amostra de 60 alunos cursando as séries iniciais do ensino fundamental.

Esses dados são prescritivos, fornecendo pistas e indicações sobre com quem e sobre o que intervir para prevenir ou limitar possíveis danos à carreira escolar e social desses alunos, os quais, no caso do grupo com dificuldades de aprendizagem escolar, estão sujeitos ao risco de fracasso e exclusão, com conseqüências emocionais e para a saúde física.

\section{Referências}

Andriola, W. B., \& Cavalcante, L. R. (1999). Avaliação da depressão infantil em alunos da pré-escola. Psicologia: Reflexão \& Crítica, 12 (2), 419-428.

Biaggio, A. (1983). Inventário de Ansiedade Traço-Estado IDATE-C: manual para a forma experimental infantil em Português. Rio de Janeiro: CEPA.

Carvalho, A. E. V., Linhares, M. B. M., \& Martinez, F. E. (2001). História de desenvolvimento e comportamentos de crianças nascidas pré-termo e baixo peso $(<1,500 \mathrm{~g})$. Psicologia: Reflexão e Crítica, 14 (1), 1-33.

Dias, M. R., Barbosa, G. A., Gaião, A. A., \& Di Lorenzo, W. F. (1997). Parâmetros psicométricos da ESDM. Infanto Revista de Neuropsiquiatria da Infância eAdolescência, 5 (1), 19-25.

Fonseca, J. S., \& Martins, G. A. (1994). Curso de estatística. São Paulo: Atlas.

Gomes, F. P. (1981). Curso de estatística experimental. São Paulo: Nobel.

Graminha, S. S. V. (1994). Problemas emocionais/comportamentais em uma amostra de escolares: incidência em função do sexo e idade. Psico, 25 (1), 49-74.

Instituto Brasileiro de Geografia e Estatística. (2002). Censo demográfico 2000: tabulação avançada. Acesso em agosto 1, 2003, disponível em http://www.ibge.org.br

Johnson, J. M., Baumgart, D., Helmstetter, E., \& Curry, C. A. (1996). Appendix A. Interview approach to a functional analysis of problem behavior. In Augmenting basic communication in natural contexts (pp.247-251). Baltimore: Paul H. Brookes Publishing.

Lipp, M. N., Souza, P. A. E., Romano, F. S. A., \& Covolan, M. A. (1991). Como enfrentar o stress infantil. São Paulo: Ícone. 
Lipp, M. N., \& Lucarelli, M. D. M. (1998). Escala de stress infantil (ESI): manual. São Paulo: Casa do Psicólogo.

Magna, J. M., \& Marturano, E. M. (1998). Atraso escolar: um levantamento de condições de desenvolvimento antecedentes à entrada na primeira série. In E. M. Marturano, A. W. Zuardi, S. R. Loureiro \& M. A. C. Figueiredo (Orgs.), Estudos de Saúde Mental (pp.9-47). Ribeirão Preto: Faculdade de Medicina de Ribeirão Preto.

Marturano, E. M. (1997). A criança, o insucesso escolar precoce e a família: condições de resiliência e vulnerabilidade. In E. M. Marturano, S. R. Loureiro \& A. W. Zuardi (Orgs.), Estudos em saúde mental (pp.130-151). Ribeirão Preto: CPG em Saúde Mental - FMRP/USP.

Marturano, E. M. (1998). Ambiente familiar e aprendizagem escolar. In C. A. Funayama (Org.), Problemas de aprendizagem: enfoque multidisciplinar (pp.73-90). Ribeirão Preto: Legis Summa.

Marturano, E. M., \& Ferreira, M. C. T. (2004). A criança com queixas escolares e sua família. In E. M. Marturano, M. B. M. Linhares \& S. R. Loureiro (Orgs.), Vulnerabilidade e proteção: indicadores na trajetória de desenvolvimento do escolar (pp.217-249). São Paulo: Casa do Psicólogo.

McKinney, J. D. (1989). Longitudinal research on the behavioral characteristics of children with learning disabilities. Journal of Learning Disabilities, 22 (3), 141-150.

Medeiros, P. C., Loureiro, S. R., Linhares, M. B. M., \& Marturano, E. M. (2000). A auto-eficácia e os aspectos comportamentais de crianças com dificuldade de aprendizagem. Psicologia: Reflexão \& Crítica, 13 (3), 327-336.

Meltzoff, J. (2001). Critical thinking about research: Psychology and related fields. Washington: American Psychological Association.

Nascimento, C. R. R. (2001). Relações entre a resposta de ansiedade de pais e mães e a resposta de ansiedade de seus filhos. Estudos de Psicologia (Campinas), 18 (2), 17-28.

Oatley, K., \& Nundy, S. (2000). Repensando o papel das emoções na Educação. In D. R. Olson \& N. Torrance
(Orgs.), Educação e desenvolvimento humano: novos modelos de aprendizagem, ensino e escolarização (pp.217-230). Porto Alegre: ArtMed.

Rutter, M. (1981). The city and the child. American Journal of Orthopsychiatry, 51 (4), 610-625.

Rutter, M. (1989). Isle of wight revisited: twenty-five years of child psychiatric epidemiology. Journal of the American Academy of Child and Adolescent Psychiatry, 28 (5), 633-653.

Santos, L. C., \& Marturano, E. M. (1999). Crianças com dificuldade de aprendizagem: um estudo de seguimento. Psicologia: Reflexão \& Crítica, 12 (2), 377-394.

Shapiro, E. S., \& Bradley, K. L. (1999). Tratamento de problemas escolares. In M. A. Reinecke, F. M. Dattilio \& A. Freeman (Orgs.), Terapia cognitiva em crianças e adolescentes: manual para a prática clínica (pp.261-276). Porto Alegre: ArtMed.

Smith, D. F. (2001). Negative emotions and coronary heart disease: causally related or merely coexistent? A review. Scandinavian Journal of Psychology, 42 (1), 57-69.

Soares, N. E., \& Fernandes, L. M. (1989). A medida do nível sócio-econômico-cultural.Arquivos Brasileiros de Psicologia, 2, 35-43.

Stein, L. M. (1994). Teste de desempenho escolar: manual para aplicação e interpretação. São Paulo: Casa do Psicólogo.

Tanganelli, M. S. L., \& Lipp, M. E. N. (1998). Sintomas de stress na rede pública de ensino. Estudos de Psicologia (Campinas), 15 (3), 17-27.

Trapani, C., \& Gettinger, M. (1999). Tratamento de alunos com transtornos de aprendizagem: Definição de caso e planejamento de programa. In M. A. Reinecke, F. M. Dattilio \& A. Freeman (Orgs.), Terapia cognitiva em crianças e adolescentes: manual para a prática clínica (pp.197-214). Porto Alegre: ArtMed.

Recebido em: 24/1/2005

Versão final reapresentada em 27/9/2005

Aprovado em: 18/10/2005 
